\title{
Determination of the Most Stable Configuration Pair(s) of the Constituent Matrix Shells and the Set of Most Stable Configurations of the Corresponding Matrix Shell System
}

Debopam Ghosh

\begin{abstract}
The present article addresses the issue of determining the most stable configuration pair(s) of a Matrix Shell, and thereby, of determining the set of most stable configurations of the associated Matrix Shell System. The problem is resolved using the criteria of spectral proximity w.r.t. the Ordered Eigenspectrum of a defined Baseline Matrix (both for Individual constituent Matrix Shells and the Matrix Shell System) and quantified in terms of an appropriate Proximity Function, the article presents the analytic expressions of the Matrix Shell Baseline elements, corresponding to $A(0,2 n), A(0,2 n-1), A(1,2 n), A(1,2 n-1)$ and $A(t, 2 n), A(t, 2 n-1)$ where $t \geq 2$, type Matrix Shells and defines the Baseline Matrices in terms of these Baseline elements, the article then provides a mathematical framework to determine the most stable Configuration pair(s) of constituent Matrix Shells and the set of most stable configurations of the Matrix Shell System and concludes with demonstration of the working of the presented framework through hypothetical examples based case studies.
\end{abstract}

Keywords:- Matrix Shell, Matrix Shell System, State-Interaction Matrix, Matrix Shell Baseline elements.

Notations

- $B(t, N)$ denotes the Baseline Matrix associated with the Matrix Shell $A(t, N)$, where $t=0,1, \ldots \ldots,(n-1)$ and $N=2 n$ or $N=2 n-1$

- $B(N)$ denotes the Baseline Matrix associated with the Matrix Shell System $N=2 n$ or $N=2 n-1$

- $S(j \mid t, N)$ denotes the State-Interaction Matrix associated with ' $\mathrm{j}^{\text {th }}$ configuration pair pertaining to the Matrix Shell $A(t, N)$, here $j=1,2, \ldots \ldots, 32$ and the indexing scheme is the same as adopted in chapter 3 , of [1]

- $S\left(j_{1}, j_{2}, \ldots \ldots, j_{n} \mid N\right)$ denotes the State-Interaction Matrix associated with the Matrix Shell System $N=2 n$ or $N=2 n-1$, where " $\mathrm{j}_{t+1}$ " denotes the index, in accordance with the indexing scheme adopted in chapter 3 of [1], of the configuration pair associated with the Matrix Shell $A(t, N)$

- $D(j \mid t, N)$ denotes the Baseline Deviation Matrix associated with the State-Interaction Matrix $S(j \mid t, N)$

- $D\left(j_{1}, j_{2}, \ldots . ., j_{n} \mid N\right)$ denotes the Baseline Deviation Matrix associated with the State-Interaction Matrix $S\left(j_{1}, j_{2}, \ldots \ldots, j_{n} \mid N\right)$

- $I_{2 \times 2}=\left[\begin{array}{ll}1 & 0 \\ 0 & 1\end{array}\right]$ and $\Sigma=\left[\begin{array}{ll}0 & 1 \\ 1 & 0\end{array}\right]$

- $O \cdot E S(B(t, N))$ denotes the Ordered Eigen spectrum of the Baseline Matrix $B(t, N)$

- $O . E S(B(N))$ denotes the Ordered Eigen spectrum of the Baseline Matrix $B(N)$

- $O . E S(S(j \mid t, N))$ denotes the Ordered Eigen spectrum of the State-Interaction Matrix $S(j \mid t, N)$

- $O . E S\left(S\left(j_{1}, j_{2}, \ldots, j_{n} \mid N\right)\right)$ denotes the Ordered Eigen spectrum of the State-Interaction Matrix $S\left(j_{1}, j_{2}, \ldots \ldots, j_{n} \mid N\right)$

- $\theta(N)$ denotes the Effective Mass of the Matrix Shell System $N=2 n$ or $N=2 n-1$

- $w(N)$ denotes the Weightage of the Matrix Shell System $N=2 n$ or $N=2 n-1$

- $I . S$ denotes the Index set associated with the Configuration pairs of the Matrix Shell $A(t, N)$

- $P\left(M_{n \times n}, N_{n \times n}\right)$ denotes the Proximity value of the Ordered Eigen spectrum of the real, symmetric matrix $M_{n \times n}$ w.r.t. the Ordered Eigen spectrum of the real, symmetric matrix $N_{n \times n}$ 
- $\left\{S\left(c_{1}, c_{2}, \ldots, c_{n} \mid N\right)\right\}$ denotes the set of all State-Interaction Matrix descriptions of the Matrix Shell System $N=2 n$ or $N=2 n-1$, that conform with satisfying the condition of Maximum Stability individually for each of the constituent Matrix Shells: $A(0, N), A(1, N), \ldots ., A(n-1, N)$

- $\left|\left\{S\left(c_{1}, c_{2}, \ldots ., c_{n} \mid N\right)\right\}\right|$ denotes the cardinality of the set $\left\{S\left(c_{1}, c_{2}, \ldots ., c_{n} \mid N\right)\right\}$

- $\left\{\left(v_{1} ; v_{2} ; \ldots, v_{n}\right)\right\}$ denotes the set of all possible Configurations of the Matrix Shell System $N=2 n$ or $N=2 n-1$, that is associated with the set $\left\{S\left(c_{1}, c_{2}, \ldots, c_{n} \mid N\right)\right\}$, here, $v_{1}$ denotes the configuration of $A(0, N)$ Matrix Shell, $v_{2}$ denotes the configuration of $A(1, N)$ Matrix Shell,.........., $v_{n}$ denotes the configuration of $A(n-1, N)$ Matrix Shell.

- $\left|\left\{\left(v_{1} ; v_{2} ; \ldots, v_{n}\right)\right\}\right|$ denotes the cardinality of the set $\left\{\left(v_{1} ; v_{2} ; \ldots, v_{n}\right)\right\}$

- $\left\{S\left(\psi_{1}, \psi_{2}, \ldots, \psi_{n} \mid N\right)\right\}$ is the subset of the set $\left\{S\left(c_{1}, c_{2}, \ldots ., c_{n} \mid N\right)\right\}$ that conform to the condition of maximum stability w.r.t. the spectral proximity criterion in terms of Matrix Shell System Baseline Matrix $B(N)$

- $\left|\left\{S\left(\psi_{1}, \psi_{2}, \ldots ., \psi_{n} \mid N\right)\right\}\right|$ denotes the cardinality of the set $\left\{S\left(\psi_{1}, \psi_{2}, \ldots, \psi_{n} \mid N\right)\right\}$

- $\left\{\left(\phi_{1} ; \phi_{2} ; \ldots . . ; \phi_{n}\right)\right\}$ denotes the set of all possible Configurations of the Matrix Shell System $N=2 n$ or $N=2 n-1$, that is associated with the set $\left\{S\left(\psi_{1}, \psi_{2}, \ldots, \psi_{n} \mid N\right)\right\}$, here, $\phi_{1}$ denotes the configuration of $A(0, N)$ Matrix Shell, $\phi_{2}$ denotes the configuration of $A(1, N)$ Matrix Shell,......., $\phi_{n}$ denotes the configuration of $A(n-1, N)$ Matrix Shell.

- $\left|\left\{\left(\phi_{1} ; \phi_{2} ; \ldots . . ; \phi_{n}\right)\right\}\right|$ denotes the cardinality of the set $\left\{\left(\phi_{1} ; \phi_{2} ; \ldots . ; \phi_{n}\right)\right\}$

\section{INTRODUCTION}

The Matrix Shell Model formalism [1] considers visualizing a finite, Complex Square Matrix as a numerical structure composed of Interpenetrating (overlapping) algebraic sub-structures termed as "Matrix Shells", a complex square matrix becomes a "Matrix Shell System" with the concept of "Directionality" incorporated at the level of the most basic unit of the Matrix Shell framework, namely, the "Basic Pathways" associated with the individual constituent Matrix Shells. In the Matrix Shell framework, the Basic Pathways compose the constituent Matrix Shells which in turn constitute the Matrix Shell System. A Matrix Shell system can be Even type $(\mathrm{N}=2 \mathrm{n}$ type) or Odd Type ( $\mathrm{N}=2 \mathrm{n}-1$ type), both of which is constituted by ' $\mathrm{n}$ ' Matrix Shells: $\mathrm{A}(0, \mathrm{~N}), \mathrm{A}(1, \mathrm{~N}) \ldots ., \mathrm{A}(\mathrm{n}-1, \mathrm{~N})$ of which some or all may be numerically active depending on the precise numerical complex matrix under consideration.

In this paper, the findings of further research studies on Matrix Shell Model framework is presented, in particular, about the analysis of State-Interaction matrix description of the Matrix Shell configurations, in [1] results on all possible Matrix Shell Configurations and their State-Interaction matrix descriptions is presented, In this research paper a mathematical scheme is proposed that determines the most stable Configuration (most preferred configuration) of a Matrix shell in a given numerical realization based on spectral proximity (quantified in terms of a Proximity Function) relative to the ordered eigenspectrum of the " Baseline Matrix" associated with the considered matrix shell, the Baseline Matrix is defined in terms of the Matrix shell baseline elements, the analytical expressions for such baseline elements for each possible type of Matrix shell is presented in this paper. Once the set of most stable configurations for each constituent Matrix Shell is determined, the resulting set of all possible configurations of the Matrix Shell system is screened, again using the condition of spectral proximity, but relative to the ordered eigenspectrum of the Baseline Matrix associated with the Matrix shell system which is constructed as a linear combination of the baseline matrices associated with individual matrix shells, to finally determine the set of most stable configurations(most preferred configurations) of the Matrix Shell system.

\section{THE ANALYTICAL EXPRESSIONS FOR THE BASELINE ELEMENTS ASSOCIATED WITH MATRIX} SHELLS

This section provides the set of Analytical Expressions pertaining to the Matrix Shell Baseline Elements, termed as the “ $\mu$ coefficients", associated with $A(0,2 n), A(0,2 n-1), A(1,2 n-1) \quad A(1,2 n)$ Matrix Shells, and for $A(t, 2 n)$ and $A(t, 2 n-1)$, where $t \geq 2$, type Matrix Shells.

Note: The notations and relevant terminologies used in this section are in accordance to that used in [1]

* $A(0,2 n)$ type Matrix Shells

- $\mu_{1}=\Gamma\left(\alpha_{1}, \alpha_{2}\right)\left(\frac{1}{2}\right)\left(\left|a_{n, n}\right|+\left|a_{n+1, n+1}\right|\right)\left(\left|a_{n, n}\right|+\left|a_{n, n+1}\right|\right)$ 
- $\mu_{2}=\Gamma\left(\alpha_{1}, \alpha_{3}\right)\left(\frac{1}{2}\right)\left(\left|a_{n, n}\right|+\left|a_{n+1, n+1}\right|\right)\left(\left|a_{n+1, n}\right|+\left|a_{n+1, n+1}\right|\right)$

- $\mu_{3}=\Gamma\left(\alpha_{2}, \alpha_{3}\right)\left(\frac{1}{2}\right)\left(\left|a_{n, n}\right|+\left|a_{n, n+1}\right|\right)\left(\left|a_{n+1, n}\right|+\left|a_{n+1, n+1}\right|\right)$

- $\mu_{4}=\Gamma\left(\beta_{1}, \beta_{2}\right)\left(\frac{1}{2}\right)\left(\left|a_{n, n+1}\right|+\left|a_{n+1, n}\right|\right)\left(\left|a_{n, n}\right|+\left|a_{n+1, n}\right|\right)$

- $\mu_{5}=\Gamma\left(\beta_{1}, \beta_{3}\right)\left(\frac{1}{2}\right)\left(\left|a_{n, n+1}\right|+\left|a_{n+1, n}\right|\right)\left(\left|a_{n, n+1}\right|+\left|a_{n+1, n+1}\right|\right)$

- $\mu_{6}=\Gamma\left(\beta_{2}, \beta_{3}\right)\left(\frac{1}{2}\right)\left(\left|a_{n, n}\right|+\left|a_{n+1, n}\right|\right)\left(\left|a_{n, n+1}\right|+\left|a_{n+1, n+1}\right|\right)$

- $\mu_{7}=\Gamma\left(\alpha_{1}, \beta_{1}\right)\left(\frac{1}{2}\right)\left(\left|a_{n, n}\right|+\left|a_{n+1, n+1}\right|\right)\left(\left|a_{n, n+1}\right|+\left|a_{n+1, n}\right|\right)$

- $\quad \mu_{8}=\Gamma\left(\alpha_{1}, \beta_{2}\right)\left(\frac{1}{2}\right)\left(\left|a_{n, n}\right|+\left|a_{n+1, n+1}\right|\right)\left(\left|a_{n, n}\right|+\left|a_{n+1, n}\right|\right)$

- $\quad \mu_{9}=\Gamma\left(\alpha_{1}, \beta_{3}\right)\left(\frac{1}{2}\right)\left(\left|a_{n, n}\right|+\left|a_{n+1, n+1}\right|\right)\left(\left|a_{n, n+1}\right|+\left|a_{n+1, n+1}\right|\right)$

- $\mu_{10}=\Gamma\left(\alpha_{2}, \beta_{1}\right)\left(\frac{1}{2}\right)\left(\left|a_{n, n}\right|+\left|a_{n, n+1}\right|\right)\left(\left|a_{n, n+1}\right|+\left|a_{n+1, n}\right|\right)$

- $\mu_{11}=\Gamma\left(\alpha_{2}, \beta_{2}\right)\left(\frac{1}{2}\right)\left(\left|a_{n, n}\right|+\left|a_{n, n+1}\right|\right)\left(\left|a_{n, n}\right|+\left|a_{n+1, n}\right|\right)$

- $\mu_{12}=\Gamma\left(\alpha_{2}, \beta_{3}\right)\left(\frac{1}{2}\right)\left(\left|a_{n, n}\right|+\left|a_{n, n+1}\right|\right)\left(\left|a_{n, n+1}\right|+\left|a_{n+1, n+1}\right|\right)$

- $\mu_{13}=\Gamma\left(\alpha_{3}, \beta_{1}\right)\left(\frac{1}{2}\right)\left(\left|a_{n+1, n}\right|+\left|a_{n+1, n+1}\right|\right)\left(\left|a_{n, n+1}\right|+\left|a_{n+1, n}\right|\right)$

- $\mu_{14}=\Gamma\left(\alpha_{3}, \beta_{2}\right)\left(\frac{1}{2}\right)\left(\left|a_{n+1, n}\right|+\left|a_{n+1, n+1}\right|\right)\left(\left|a_{n, n}\right|+\left|a_{n+1, n}\right|\right)$

- $\mu_{15}=\Gamma\left(\alpha_{3}, \beta_{3}\right)\left(\frac{1}{2}\right)\left(\left|a_{n+1, n}\right|+\left|a_{n+1, n+1}\right|\right)\left(\left|a_{n, n+1}\right|+\left|a_{n+1, n+1}\right|\right)$

* $A(0,2 n-1)$ type Matrix Shells

We have, from [1], the following expressions pertaining to $A(0,2 n-1)$ type Matrix Shells:

$>|X|=\left(\frac{1}{4}\right)\left|a_{n, n}\right|$

$>\hat{X}=2|X|(1-\exp (-|X|))$

Therefore, the analytical expressions for the baseline elements are as following:

- $\mu_{1}=\mu_{2}=\mu_{4}=\mu_{5}=\mu_{P}$

- $\mu_{P}=\left[\left(\frac{1}{4}\right)(1-\exp (-2 \hat{X}))(1-\exp (-\hat{X}))^{2}+\left(\frac{3}{4}\right)(1-\exp (-2 \hat{X}))^{5}\right]\left(\frac{1}{8}\right)\left|a_{n, n}\right|^{2}$

- $\mu_{3}=\mu_{6}=\mu_{Q}$ 
- $\mu_{Q}=(1-\exp (-2 \hat{X}))^{5}\left(\frac{1}{8}\right)\left|a_{n, n}\right|^{2}$

- $\mu_{7}=\mu o$

- $\mu o=(1-\exp (-2 \hat{X}))^{7}\left(\frac{1}{8}\right)\left|a_{n, n}\right|^{2}$

- $\mu_{8}=\mu_{9}=\mu_{10}=\mu_{11}=\mu_{12}=\mu_{13}=\mu_{14}=\mu_{15}=\mu_{N}$

- $\mu_{N}=\left[\left(\frac{1}{4}\right)(1-\exp (-2 \hat{X}))^{3}(1-\exp (-\hat{X}))^{2}+\left(\frac{3}{4}\right)(1-\exp (-2 \hat{X}))^{7}\right]\left(\frac{1}{8}\right)\left|a_{n, n}\right|^{2}$

\section{* $A(1,2 n-1)$ type Matrix Shells}

- $\mu_{1}=\Gamma\left(\alpha_{1}, \alpha_{2}\right)\left[\left(\left(\frac{1}{2}\right)\left(\left|a_{n-1, n-1}\right|+\left|a_{n+1, n+1}\right|\right)\left(\left|a_{n-1, n-1}\right|+\left|a_{n-1, n+1}\right|\right)\right)+\left(\left|a_{n, n}\right|\left|a_{n-1, n}\right|\right)\right]$

- $\mu_{2}=\Gamma\left(\alpha_{1}, \alpha_{3}\right)\left[\left(\left(\frac{1}{2}\right)\left(\left|a_{n-1, n-1}\right|+\left|a_{n+1, n+1}\right|\right)\left(\left|a_{n+1, n-1}\right|+\left|a_{n+1, n+1}\right|\right)\right)+\left(\left|a_{n, n}\right|\left|a_{n+1, n}\right|\right)\right]$

- $\mu_{3}=\Gamma\left(\alpha_{2}, \alpha_{3}\right)\left[\left(\left(\frac{1}{2}\right)\left(\left|a_{n-1, n-1}\right|+\left|a_{n-1, n+1}\right|\right)\left(\left|a_{n+1, n-1}\right|+\left|a_{n+1, n+1}\right|\right)\right)+\left(\left|a_{n-1, n}\right|\left|a_{n+1, n}\right|\right)\right]$

- $\mu_{4}=\Gamma\left(\beta_{1}, \beta_{2}\right)\left[\left(\left(\frac{1}{2}\right)\left(\left|a_{n-1, n+1}\right|+\left|a_{n+1, n-1}\right|\right)\left(\left|a_{n-1, n-1}\right|+\left|a_{n+1, n-1}\right|\right)\right)+\left(\left|a_{n, n}\right|\left|a_{n, n-1}\right|\right)\right]$

- $\mu_{5}=\Gamma\left(\beta_{1}, \beta_{3}\right)\left[\left(\left(\frac{1}{2}\right)\left(\left|a_{n-1, n+1}\right|+\left|a_{n+1, n-1}\right|\right)\left(\left|a_{n-1, n+1}\right|+\left|a_{n+1, n+1}\right|\right)\right)+\left(\left|a_{n, n}\right|\left|a_{n, n+1}\right|\right)\right]$

- $\mu_{6}=\Gamma\left(\beta_{2,}, \beta_{3}\right)\left[\left(\left(\frac{1}{2}\right)\left(\left|a_{n-1, n-1}\right|+\left|a_{n+1, n-1}\right|\right)\left(\left|a_{n-1, n+1}\right|+\left|a_{n+1, n+1}\right|\right)\right)+\left(\left|a_{n, n-1}\right|\left|a_{n, n+1}\right|\right)\right]$

- $\mu_{7}=\Gamma\left(\alpha_{1}, \beta_{1}\right)\left[\left(\left(\frac{1}{2}\right)\left(\left|a_{n-1, n-1}\right|+\left|a_{n+1, n+1}\right|\right)\left(\left|a_{n-1, n+1}\right|+\left|a_{n+1, n-1}\right|\right)\right)+\left(\left|a_{n, n}\right|^{2}\right)\right]$

- $\mu_{8}=\Gamma\left(\alpha_{1}, \beta_{2}\right)\left[\left(\left(\frac{1}{2}\right)\left(\left|a_{n-1, n-1}\right|+\left|a_{n+1, n+1}\right|\right)\left(\left|a_{n-1, n-1}\right|+\left|a_{n+1, n-1}\right|\right)\right)+\left(\left|a_{n, n}\right|\left|a_{n, n-1}\right|\right)\right]$

- $\mu_{9}=\Gamma\left(\alpha_{1}, \beta_{3}\right)\left[\left(\left(\frac{1}{2}\right)\left(\left|a_{n-1, n-1}\right|+\left|a_{n+1, n+1}\right|\right)\left(\left|a_{n-1, n+1}\right|+\left|a_{n+1, n+1}\right|\right)\right)+\left(\left|a_{n, n}\right|\left|a_{n, n+1}\right|\right)\right]$

- $\mu_{10}=\Gamma\left(\alpha_{2}, \beta_{1}\right)\left[\left(\left(\frac{1}{2}\right)\left(\left|a_{n-1, n-1}\right|+\left|a_{n-1, n+1}\right|\right)\left(\left|a_{n-1, n+1}\right|+\left|a_{n+1, n-1}\right|\right)\right)+\left(\left|a_{n-1, n}\right|\left|a_{n, n}\right|\right)\right]$

- $\mu_{11}=\Gamma\left(\alpha_{2}, \beta_{2}\right)\left[\left(\left(\frac{1}{2}\right)\left(\left|a_{n-1, n-1}\right|+\left|a_{n-1, n+1}\right|\right)\left(\left|a_{n-1, n-1}\right|+\left|a_{n+1, n-1}\right|\right)\right)+\left(\left|a_{n-1, n}\right|\left|a_{n, n-1}\right|\right)\right]$

- $\mu_{12}=\Gamma\left(\alpha_{2}, \beta_{3}\right)\left[\left(\left(\frac{1}{2}\right)\left(\left|a_{n-1, n-1}\right|+\left|a_{n-1, n+1}\right|\right)\left(\left|a_{n-1, n+1}\right|+\left|a_{n+1, n+1}\right|\right)\right)+\left(\left|a_{n-1, n}\right|\left|a_{n, n+1}\right|\right)\right]$

- $\mu_{13}=\Gamma\left(\alpha_{3}, \beta_{1}\right)\left[\left(\left(\frac{1}{2}\right)\left(\left|a_{n+1, n-1}\right|+\left|a_{n+1, n+1}\right|\right)\left(\left|a_{n-1, n+1}\right|+\left|a_{n+1, n-1}\right|\right)\right)+\left(\left|a_{n+1, n}\right|\left|a_{n, n}\right|\right)\right]$ 
- $\mu_{14}=\Gamma\left(\alpha_{3}, \beta_{2}\right)\left[\left(\left(\frac{1}{2}\right)\left(\left|a_{n+1, n-1}\right|+\left|a_{n+1, n+1}\right|\right)\left(\left|a_{n-1, n-1}\right|+\left|a_{n+1, n-1}\right|\right)\right)+\left(\left|a_{n+1, n}\right|\left|a_{n, n-1}\right|\right)\right]$

- $\mu_{15}=\Gamma\left(\alpha_{3}, \beta_{3}\right)\left[\left(\left(\frac{1}{2}\right)\left(\left|a_{n+1, n-1}\right|+\left|a_{n+1, n+1}\right|\right)\left(\left|a_{n-1, n+1}\right|+\left|a_{n+1, n+1}\right|\right)\right)+\left(\left|a_{n+1, n}\right|\left|a_{n, n+1}\right|\right)\right]$

\section{* $A(1,2 n)$ type Matrix Shells}

- $\mu_{1}=\Gamma\left(\alpha_{1}, \alpha_{2}\right)\left(\frac{1}{2}\right)\left[\left(\left(\left|a_{n-1, n-1}\right|+\left|a_{n+2, n+2}\right|\right)\left(\left|a_{n-1, n-1}\right|+\left|a_{n-1, n+2}\right|\right)\right)+\left(\left(\left|a_{n, n}\right|+\left|a_{n+1, n+1}\right|\right)\left(\left|a_{n-1, n}\right|+\left|a_{n-1, n+1}\right|\right)\right)\right]$

- $\mu_{2}=\Gamma\left(\alpha_{1}, \alpha_{3}\right)\left(\frac{1}{2}\right)\left[\left(\left(\left|a_{n-1, n-1}\right|+\left|a_{n+2, n+2}\right|\right)\left(\left|a_{n+2, n-1}\right|+\left|a_{n+2, n+2}\right|\right)\right)+\left(\left(\left|a_{n, n}\right|+\left|a_{n+1, n+1}\right|\right)\left(\left|a_{n+2, n}\right|+\left|a_{n+2, n+1}\right|\right)\right)\right]$

- $\mu_{3}=\Gamma\left(\alpha_{2}, \alpha_{3}\right)\left(\frac{1}{2}\right)\left[\left(\left(\left|a_{n-1, n-1}\right|+\left|a_{n-1, n+2}\right|\right)\left(\left|a_{n+2, n-1}\right|+\left|a_{n+2, n+2}\right|\right)\right)+\left(\left(\left|a_{n-1, n}\right|+\left|a_{n-1, n+1}\right|\right)\left(\left|a_{n+2, n}\right|+\left|a_{n+2, n+1}\right|\right)\right)\right]$

- $\mu_{4}=\Gamma\left(\beta_{1}, \beta_{2}\right)\left(\frac{1}{2}\right)\left[\left(\left(\left|a_{n-1, n+2}\right|+\left|a_{n+2, n-1}\right|\right)\left(\left|a_{n-1, n-1}\right|+\left|a_{n+2, n-1}\right|\right)\right)+\left(\left(\left|a_{n, n+1}\right|+\left|a_{n+1, n}\right|\right)\left(\left|a_{n, n-1}\right|+\left|a_{n+1, n-1}\right|\right)\right)\right]$

- $\mu_{5}=\Gamma\left(\beta_{1}, \beta_{3}\right)\left(\frac{1}{2}\right)\left[\left(\left(\left|a_{n-1, n+2}\right|+\left|a_{n+2, n-1}\right|\right)\left(\left|a_{n-1, n+2}\right|+\left|a_{n+2, n+2}\right|\right)\right)+\left(\left(\left|a_{n, n+1}\right|+\left|a_{n+1, n}\right|\right)\left(\left|a_{n, n+2}\right|+\left|a_{n+1, n+2}\right|\right)\right)\right]$

- $\mu_{6}=\Gamma\left(\beta_{2,}, \beta_{3}\right)\left(\frac{1}{2}\right)\left[\left(\left(\left|a_{n-1, n-1}\right|+\left|a_{n+2, n-1}\right|\right)\left(\left|a_{n-1, n+2}\right|+\left|a_{n+2, n+2}\right|\right)\right)+\left(\left(\left|a_{n, n-1}\right|+\left|a_{n+1, n-1}\right|\right)\left(\left|a_{n, n+2}\right|+\left|a_{n+1, n+2}\right|\right)\right)\right]$

- $\mu_{7}=\Gamma\left(\alpha_{1}, \beta_{1}\right)\left(\frac{1}{2}\right)\left[\left(\left(\left|a_{n-1, n-1}\right|+\left|a_{n+2, n+2}\right|\right)\left(\left|a_{n-1, n+2}\right|+\left|a_{n+2, n-1}\right|\right)\right)+\left(\left(\left|a_{n, n}\right|+\left|a_{n+1, n+1}\right|\right)\left(\left|a_{n, n+1}\right|+\left|a_{n+1, n}\right|\right)\right)\right]$

- $\mu_{8}=\Gamma\left(\alpha_{1}, \beta_{2}\right)\left(\frac{1}{2}\right)\left[\left(\left(\left|a_{n-1, n-1}\right|+\left|a_{n+2, n+2}\right|\right)\left(\left|a_{n-1, n-1}\right|+\left|a_{n+2, n-1}\right|\right)\right)+\left(\left(\left|a_{n, n}\right|+\left|a_{n+1, n+1}\right|\right)\left(\left|a_{n, n-1}\right|+\left|a_{n+1, n-1}\right|\right)\right)\right]$

- $\mu_{9}=\Gamma\left(\alpha_{1}, \beta_{3}\right)\left(\frac{1}{2}\right)\left[\left(\left(\left|a_{n-1, n-1}\right|+\left|a_{n+2, n+2}\right|\right)\left(\left|a_{n-1, n+2}\right|+\left|a_{n+2, n+2}\right|\right)\right)+\left(\left(\left|a_{n, n}\right|+\left|a_{n+1, n+1}\right|\right)\left(\left|a_{n, n+2}\right|+\left|a_{n+1, n+2}\right|\right)\right)\right]$

- $\mu_{10}=\Gamma\left(\alpha_{2}, \beta_{1}\right)\left(\frac{1}{2}\right)\left[\left(\left(\left|a_{n-1, n-1}\right|+\left|a_{n-1, n+2}\right|\right)\left(\left|a_{n-1, n+2}\right|+\left|a_{n+2, n-1}\right|\right)\right)+\left(\left(\left|a_{n-1, n}\right|+\left|a_{n-1, n+1}\right|\right)\left(\left|a_{n, n+1}\right|+\left|a_{n+1, n}\right|\right)\right)\right]$

- $\mu_{11}=\Gamma\left(\alpha_{2}, \beta_{2}\right)\left(\frac{1}{2}\right)\left[\left(\left(\left|a_{n-1, n-1}\right|+\left|a_{n-1, n+2}\right|\right)\left(\left|a_{n-1, n-1}\right|+\left|a_{n+2, n-1}\right|\right)\right)+\left(\left(\left|a_{n-1, n}\right|+\left|a_{n-1, n+1}\right|\right)\left(\left|a_{n, n-1}\right|+\left|a_{n+1, n-1}\right|\right)\right)\right]$

- $\mu_{12}=\Gamma\left(\alpha_{2}, \beta_{3}\right)\left(\frac{1}{2}\right)\left[\left(\left(\left|a_{n-1, n-1}\right|+\left|a_{n-1, n+2}\right|\right)\left(\left|a_{n-1, n+2}\right|+\left|a_{n+2, n+2}\right|\right)\right)+\left(\left(\left|a_{n-1, n}\right|+\left|a_{n-1, n+1}\right|\right)\left(\left|a_{n, n+2}\right|+\left|a_{n+1, n+2}\right|\right)\right)\right]$

- $\mu_{13}=\Gamma\left(\alpha_{3}, \beta_{1}\right)\left(\frac{1}{2}\right)\left[\left(\left(\left|a_{n+2, n-1}\right|+\left|a_{n+2, n+2}\right|\right)\left(\left|a_{n-1, n+2}\right|+\left|a_{n+2, n-1}\right|\right)\right)+\left(\left(\left|a_{n+2, n}\right|+\left|a_{n+2, n+1}\right|\right)\left(\left|a_{n, n+1}\right|+\left|a_{n+1, n}\right|\right)\right)\right]$

- $\mu_{14}=\Gamma\left(\alpha_{3}, \beta_{2}\right)\left(\frac{1}{2}\right)\left[\left(\left(\left|a_{n+2, n-1}\right|+\left|a_{n+2, n+2}\right|\right)\left(\left|a_{n-1, n-1}\right|+\left|a_{n+2, n-1}\right|\right)\right)+\left(\left(\left|a_{n+2, n}\right|+\left|a_{n+2, n+1}\right|\right)\left(\left|a_{n, n-1}\right|+\left|a_{n+1, n-1}\right|\right)\right)\right]$

- $\mu_{15}=\Gamma\left(\alpha_{3}, \beta_{3}\right)\left(\frac{1}{2}\right)\left[\left(\left(\left|a_{n+2, n-1}\right|+\left|a_{n+2, n+2}\right|\right)\left(\left|a_{n-1, n+2}\right|+\left|a_{n+2, n+2}\right|\right)\right)+\left(\left(\left|a_{n+2, n}\right|+\left|a_{n+2, n+1}\right|\right)\left(\left|a_{n, n+2}\right|+\left|a_{n+1, n+2}\right|\right)\right)\right]$

\section{* $A(t, 2 n), t \geq 2$ type Matrix Shells}

- $\mu_{1}=\Gamma\left(\alpha_{1}, \alpha_{2}\right)\left(\frac{1}{2}\right)\left[\left(\left(\left|d_{1}\right|+\left|d_{4}\right|\right)\left(\left|d_{1}\right|+\left|d_{2}\right|\right)\right)+\left(\sum_{j=1}^{t}\left(\left|s_{j}\right|+\left|s_{2 t+1-j}\right|\right)\left(\left|u_{j}\right|+\left|u_{2 t+1-j}\right|\right)\right)\right]$

- $\mu_{2}=\Gamma\left(\alpha_{1}, \alpha_{3}\right)\left(\frac{1}{2}\right)\left[\left(\left(\left|d_{1}\right|+\left|d_{4}\right|\right)\left(\left|d_{3}\right|+\left|d_{4}\right|\right)\right)+\left(\sum_{j=1}^{t}\left(\left|s_{j}\right|+\left|s_{2 t+1-j}\right|\right)\left(\left|v_{j}\right|+\left|v_{2 t+1-j}\right|\right)\right)\right]$ 
- $\mu_{3}=\Gamma\left(\alpha_{2}, \alpha_{3}\right)\left(\frac{1}{2}\right)\left[\left(\left(\left|d_{1}\right|+\left|d_{2}\right|\right)\left(\left|d_{3}\right|+\left|d_{4}\right|\right)\right)+\left(\sum_{j=1}^{t}\left(\left|u_{j}\right|+\left|u_{2 t+1-j}\right|\right)\left(\left|v_{j}\right|+\left|v_{2 t+1-j}\right|\right)\right)\right]$

- $\mu_{4}=\Gamma\left(\beta_{1}, \beta_{2}\right)\left(\frac{1}{2}\right)\left[\left(\left(\left|d_{2}\right|+\left|d_{3}\right|\right)\left(\left|d_{1}\right|+\left|d_{3}\right|\right)\right)+\left(\sum_{j=1}^{t}\left(\left|r_{j}\right|+\left|r_{2 t+1-j}\right|\right)\left(\left|p_{j}\right|+\left|p_{2 t+1-j}\right|\right)\right)\right]$

- $\mu_{5}=\Gamma\left(\beta_{1}, \beta_{3}\right)\left(\frac{1}{2}\right)\left[\left(\left(\left|d_{2}\right|+\left|d_{3}\right|\right)\left(\left|d_{2}\right|+\left|d_{4}\right|\right)\right)+\left(\sum_{j=1}^{t}\left(\left|r_{j}\right|+\left|r_{2 t+1-j}\right|\right)\left(\left|q_{j}\right|+\left|q_{2 t+1-j}\right|\right)\right)\right]$

- $\mu_{6}=\Gamma\left(\beta_{2}, \beta_{3}\right)\left(\frac{1}{2}\right)\left[\left(\left(\left|d_{1}\right|+\left|d_{3}\right|\right)\left(\left|d_{2}\right|+\left|d_{4}\right|\right)\right)+\left(\sum_{j=1}^{t}\left(\left|p_{j}\right|+\left|p_{2 t+1-j}\right|\right)\left(\left|q_{j}\right|+\left|q_{2 t+1-j}\right|\right)\right)\right]$

- $\mu_{7}=\Gamma\left(\alpha_{1}, \beta_{1}\right)\left(\frac{1}{2}\right)\left[\left(\left(\left|d_{1}\right|+\left|d_{4}\right|\right)\left(\left|d_{2}\right|+\left|d_{3}\right|\right)\right)+\left(\sum_{j=1}^{t}\left(\left|s_{j}\right|+\left|s_{2 t+1-j}\right|\right)\left(\left|r_{j}\right|+\left|r_{2 t+1-j}\right|\right)\right)\right]$

- $\mu_{8}=\Gamma\left(\alpha_{1}, \beta_{2}\right)\left(\frac{1}{2}\right)\left[\left(\left(\left|d_{1}\right|+\left|d_{4}\right|\right)\left(\left|d_{1}\right|+\left|d_{3}\right|\right)\right)+\left(\sum_{j=1}^{t}\left(\left|s_{j}\right|+\left|s_{2 t+1-j}\right|\right)\left(\left|p_{j}\right|+\left|p_{2 t+1-j}\right|\right)\right)\right]$

- $\mu_{9}=\Gamma\left(\alpha_{1}, \beta_{3}\right)\left(\frac{1}{2}\right)\left[\left(\left(\left|d_{1}\right|+\left|d_{4}\right|\right)\left(\left|d_{2}\right|+\left|d_{4}\right|\right)\right)+\left(\sum_{j=1}^{t}\left(\left|s_{j}\right|+\left|s_{2 t+1-j}\right|\right)\left(\left|q_{j}\right|+\left|q_{2 t+1-j}\right|\right)\right)\right]$

- $\mu_{10}=\Gamma\left(\alpha_{2}, \beta_{1}\right)\left(\frac{1}{2}\right)\left[\left(\left(\left|d_{1}\right|+\left|d_{2}\right|\right)\left(\left|d_{2}\right|+\left|d_{3}\right|\right)\right)+\left(\sum_{j=1}^{t}\left(\left|u_{j}\right|+\left|u_{2 t+1-j}\right|\right)\left(\left|r_{j}\right|+\left|r_{2 t+1-j}\right|\right)\right)\right]$

- $\mu_{11}=\Gamma\left(\alpha_{2}, \beta_{2}\right)\left(\frac{1}{2}\right)\left[\left(\left(\left|d_{1}\right|+\left|d_{2}\right|\right)\left(\left|d_{1}\right|+\left|d_{3}\right|\right)\right)+\left(\sum_{j=1}^{t}\left(\left|u_{j}\right|+\left|u_{2 t+1-j}\right|\right)\left(\left|p_{j}\right|+\left|p_{2 t+1-j}\right|\right)\right)\right]$

- $\mu_{12}=\Gamma\left(\alpha_{2}, \beta_{3}\right)\left(\frac{1}{2}\right)\left[\left(\left(\left|d_{1}\right|+\left|d_{2}\right|\right)\left(\left|d_{2}\right|+\left|d_{4}\right|\right)\right)+\left(\sum_{j=1}^{t}\left(\left|u_{j}\right|+\left|u_{2 t+1-j}\right|\right)\left(\left|q_{j}\right|+\left|q_{2 t+1-j}\right|\right)\right)\right]$

- $\mu_{13}=\Gamma\left(\alpha_{3}, \beta_{1}\right)\left(\frac{1}{2}\right)\left[\left(\left(\left|d_{3}\right|+\left|d_{4}\right|\right)\left(\left|d_{2}\right|+\left|d_{3}\right|\right)\right)+\left(\sum_{j=1}^{t}\left(\left|v_{j}\right|+\left|v_{2 t+1-j}\right|\right)\left(\left|r_{j}\right|+\left|r_{2 t+1-j}\right|\right)\right)\right]$

- $\mu_{14}=\Gamma\left(\alpha_{3}, \beta_{2}\right)\left(\frac{1}{2}\right)\left[\left(\left(\left|d_{3}\right|+\left|d_{4}\right|\right)\left(\left|d_{1}\right|+\left|d_{3}\right|\right)\right)+\left(\sum_{j=1}^{t}\left(\left|v_{j}\right|+\left|v_{2 t+1-j}\right|\right)\left(\left|p_{j}\right|+\left|p_{2 t+1-j}\right|\right)\right)\right]$

- $\mu_{15}=\Gamma\left(\alpha_{3}, \beta_{3}\right)\left(\frac{1}{2}\right)\left[\left(\left(\left|d_{3}\right|+\left|d_{4}\right|\right)\left(\left|d_{2}\right|+\left|d_{4}\right|\right)\right)+\left(\sum_{j=1}^{t}\left(\left|v_{j}\right|+\left|v_{2 t+1-j}\right|\right)\left(\left|q_{j}\right|+\left|q_{2 t+1-j}\right|\right)\right)\right]$

\section{* $A(t, 2 n-1), t \geq 2$ type Matrix Shells}

- $\mu_{1}=\Gamma\left(\alpha_{1}, \alpha_{2}\right)\left[\left(\frac{1}{2}\right)\left(\left(\left|d_{1}\right|+\left|d_{4}\right|\right)\left(\left|d_{1}\right|+\left|d_{2}\right|\right)+\sum_{j=1}^{t-1}\left(\left|s_{j}\right|+\left|s_{2 t}-j\right|\right)\left(\left|u_{j}\right|+\left|u_{2 t}-j\right|\right)\right)+\left(\left|c_{0}\right|\left|u_{t}\right|\right)\right]$

- $\mu_{2}=\Gamma\left(\alpha_{1}, \alpha_{3}\right)\left[\left(\frac{1}{2}\right)\left(\left(\left|d_{1}\right|+\left|d_{4}\right|\right)\left(\left|d_{3}\right|+\left|d_{4}\right|\right)+\sum_{j=1}^{t-1}\left(\left|s_{j}\right|+\left|s_{2 t-j}\right|\right)\left(\left|v_{j}\right|+\left|v_{2 t}-j\right|\right)\right)+\left(\left|c_{0}\right|\left|v_{t}\right|\right)\right]$

- $\mu_{3}=\Gamma\left(\alpha_{2}, \alpha_{3}\right)\left[\left(\frac{1}{2}\right)\left(\left(\left|d_{1}\right|+\left|d_{2}\right|\right)\left(\left|d_{3}\right|+\left|d_{4}\right|\right)+\sum_{j=1}^{t-1}\left(\left|u_{j}\right|+\left|u_{2 t}-j\right|\right)\left(\left|v_{j}\right|+\left|v_{2 t-j}\right|\right)\right)+\left(\left|u_{t}\right|\left|v_{t}\right|\right)\right]$ 
- $\mu_{4}=\Gamma\left(\beta_{1}, \beta_{2}\right)\left[\left(\frac{1}{2}\right)\left(\left(\left|d_{2}\right|+\left|d_{3}\right|\right)\left(\left|d_{1}\right|+\left|d_{3}\right|\right)+\sum_{j=1}^{t-1}\left(\left|r_{j}\right|+\left|r_{2 t-j}\right|\right)\left(\left|p_{j}\right|+\left|p_{2 t-j}\right|\right)\right)+\left(\left|c_{0}\right|\left|p_{t}\right|\right)\right]$

- $\mu_{5}=\Gamma\left(\beta_{1}, \beta_{3}\right)\left[\left(\frac{1}{2}\right)\left(\left(\left|d_{2}\right|+\left|d_{3}\right|\right)\left(\left|d_{2}\right|+\left|d_{4}\right|\right)+\sum_{j=1}^{t-1}\left(\left|r_{j}\right|+\left|r_{2 t-j}\right|\right)\left(\left|q_{j}\right|+\left|q_{2 t-j}\right|\right)\right)+\left(\left|c_{0}\right|\left|q_{t}\right|\right)\right]$

- $\mu_{6}=\Gamma\left(\beta_{2}, \beta_{3}\right)\left[\left(\frac{1}{2}\right)\left(\left(\left|d_{1}\right|+\left|d_{3}\right|\right)\left(\left|d_{2}\right|+\left|d_{4}\right|\right)+\sum_{j=1}^{t-1}\left(\left|p_{j}\right|+\left|p_{2 t-j}\right|\right)\left(\left|q_{j}\right|+\left|q_{2 t-j}\right|\right)\right)+\left(\left|p_{t}\right|\left|q_{t}\right|\right)\right]$

- $\mu_{7}=\Gamma\left(\alpha_{1}, \beta_{1}\right)\left[\left(\frac{1}{2}\right)\left(\left(\left|d_{1}\right|+\left|d_{4}\right|\right)\left(\left|d_{2}\right|+\left|d_{3}\right|\right)+\sum_{j=1}^{t-1}\left(\left|s_{j}\right|+\left|s_{2 t-j}\right|\right)\left(\left|r_{j}\right|+\left|r_{2 t-j}\right|\right)\right)+\left(\left|c_{0}\right|^{2}\right)\right]$

- $\mu_{8}=\Gamma\left(\alpha_{1}, \beta_{2}\right)\left[\left(\frac{1}{2}\right)\left(\left(\left|d_{1}\right|+\left|d_{4}\right|\right)\left(\left|d_{1}\right|+\left|d_{3}\right|\right)+\sum_{j=1}^{t-1}\left(\left|s_{j}\right|+\left|s_{2 t-j}\right|\right)\left(\left|p_{j}\right|+\left|p_{2 t-j}\right|\right)\right)+\left(\left|c_{0}\right|\left|p_{t}\right|\right)\right]$

- $\mu_{9}=\Gamma\left(\alpha_{1}, \beta_{3}\right)\left[\left(\frac{1}{2}\right)\left(\left(\left|d_{1}\right|+\left|d_{4}\right|\right)\left(\left|d_{2}\right|+\left|d_{4}\right|\right)+\sum_{j=1}^{t-1}\left(\left|s_{j}\right|+\left|s_{2 t-j}\right|\right)\left(\left|q_{j}\right|+\left|q_{2 t-j}\right|\right)\right)+\left(\left|c_{0}\right|\left|q_{t}\right|\right)\right]$

- $\mu_{10}=\Gamma\left(\alpha_{2}, \beta_{1}\right)\left[\left(\frac{1}{2}\right)\left(\left(\left|d_{1}\right|+\left|d_{2}\right|\right)\left(\left|d_{2}\right|+\left|d_{3}\right|\right)+\sum_{j=1}^{t-1}\left(\left|u_{j}\right|+\left|u_{2 t-j}\right|\right)\left(\left|r_{j}\right|+\left|r_{2 t-j}\right|\right)\right)+\left(\left|u_{t}\right|\left|c_{0}\right|\right)\right]$

- $\mu_{11}=\Gamma\left(\alpha_{2}, \beta_{2}\right)\left[\left(\frac{1}{2}\right)\left(\left(\left|d_{1}\right|+\left|d_{2}\right|\right)\left(\left|d_{1}\right|+\left|d_{3}\right|\right)+\sum_{j=1}^{t-1}\left(\left|u_{j}\right|+\left|u_{2 t-j}\right|\right)\left(\left|p_{j}\right|+\left|p_{2 t-j}\right|\right)\right)+\left(\left|u_{t}\right|\left|p_{t}\right|\right)\right]$

- $\mu_{12}=\Gamma\left(\alpha_{2}, \beta_{3}\right)\left[\left(\frac{1}{2}\right)\left(\left(\left|d_{1}\right|+\left|d_{2}\right|\right)\left(\left|d_{2}\right|+\left|d_{4}\right|\right)+\sum_{j=1}^{t-1}\left(\left|u_{j}\right|+\left|u_{2 t-j}\right|\right)\left(\left|q_{j}\right|+\left|q_{2 t-j}\right|\right)\right)+\left(\left|u_{t}\right|\left|q_{t}\right|\right)\right]$

- $\mu_{13}=\Gamma\left(\alpha_{3}, \beta_{1}\right)\left[\left(\frac{1}{2}\right)\left(\left(\left|d_{3}\right|+\left|d_{4}\right|\right)\left(\left|d_{2}\right|+\left|d_{3}\right|\right)+\sum_{j=1}^{t-1}\left(\left|v_{j}\right|+\left|v_{2 t-j}\right|\right)\left(\left|r_{j}\right|+\left|r_{2 t-j}\right|\right)\right)+\left(\left|v_{t}\right|\left|c_{0}\right|\right)\right]$

- $\mu_{14}=\Gamma\left(\alpha_{3}, \beta_{2}\right)\left[\left(\frac{1}{2}\right)\left(\left(\left|d_{3}\right|+\left|d_{4}\right|\right)\left(\left|d_{1}\right|+\left|d_{3}\right|\right)+\sum_{j=1}^{t-1}\left(\left|v_{j}\right|+\left|v_{2 t-j}\right|\right)\left(\left|p_{j}\right|+\left|p_{2 t-j}\right|\right)\right)+\left(\left|v_{t}\right|\left|p_{t}\right|\right)\right]$

- $\mu_{15}=\Gamma\left(\alpha_{3}, \beta_{3}\right)\left[\left(\frac{1}{2}\right)\left(\left(\left|d_{3}\right|+\left|d_{4}\right|\right)\left(\left|d_{2}\right|+\left|d_{4}\right|\right)+\sum_{j=1}^{t-1}\left(\left|v_{j}\right|+\left|v_{2 t-j}\right|\right)\left(\left|q_{j}\right|+\left|q_{2 t-j}\right|\right)\right)+\left(\left|v_{t}\right|\left|q_{t}\right|\right)\right]$ 

Matrix

The Analytical Expression for the Baseline Matrix and that of the State-Interaction matrices in terms of the Baseline $B(t, N)=\left[\begin{array}{cccccccccccc}\Delta_{1} & \Delta_{1} & x_{1} & \mu_{1} & x_{2} & \mu_{2} & x_{7} & \mu_{7} & x_{8} & \mu_{8} & x_{9} & \mu_{9} \\ \Delta_{1} & \Delta_{1} & \mu_{1} & x_{1} & \mu_{2} & x_{2} & \mu_{7} & x_{7} & \mu_{8} & x_{8} & \mu_{9} & x_{9} \\ x_{1} & \mu_{1} & \Delta_{2} & \Delta_{2} & x_{3} & \mu_{3} & x_{10} & \mu_{10} & x_{11} & \mu_{11} & x_{12} & \mu_{12} \\ \mu_{1} & x_{1} & \Delta_{2} & \Delta_{2} & \mu_{3} & x_{3} & \mu_{10} & x_{10} & \mu_{11} & x_{11} & \mu_{12} & x_{12} \\ x_{2} & \mu_{2} & x_{3} & \mu_{3} & \Delta_{3} & \Delta_{3} & x_{13} & \mu_{13} & x_{14} & \mu_{14} & x_{15} & \mu_{15} \\ \mu_{2} & x_{2} & \mu_{3} & x_{3} & \Delta_{3} & \Delta_{3} & \mu_{13} & x_{13} & \mu_{14} & x_{14} & \mu_{15} & x_{15} \\ x_{7} & \mu_{7} & x_{10} & \mu_{10} & x_{13} & \mu_{13} & \Delta_{4} & \Delta_{4} & x_{4} & \mu_{4} & x_{5} & \mu_{5} \\ \mu_{7} & x_{7} & \mu_{10} & x_{10} & \mu_{13} & x_{13} & \Delta_{4} & \Delta_{4} & \mu_{4} & x_{4} & \mu_{5} & x_{5} \\ x_{8} & \mu_{8} & x_{11} & \mu_{11} & x_{14} & \mu_{14} & x_{4} & \mu_{4} & \Delta_{5} & \Delta_{5} & x_{6} & \mu_{6} \\ \mu_{8} & x_{8} & \mu_{11} & x_{11} & \mu_{14} & x_{14} & \mu_{4} & x_{4} & \Delta_{5} & \Delta_{5} & \mu_{6} & x_{6} \\ x_{9} & \mu_{9} & x_{12} & \mu_{12} & x_{15} & \mu_{15} & x_{5} & \mu_{5} & x_{6} & \mu_{6} & \Delta_{6} & \Delta_{6} \\ \mu_{9} & x_{9} & \mu_{12} & x_{12} & \mu_{15} & x_{15} & \mu_{5} & x_{5} & \mu_{6} & x_{6} & \Delta_{6} & \Delta_{6}\end{array}\right]_{12 \times 12}$

$B(t, N)=\left(X(t, N) \otimes I_{2 \times 2}\right)+\left(\Lambda(t, N) \otimes \Sigma_{2 \times 2}\right)$, here, $X(t, N)$, as given in [1], has the following analytical expression:

$$
X(t, N)=\left[\begin{array}{cccccc}
\Delta_{1} & x_{1} & x_{2} & x_{7} & x_{8} & x_{9} \\
x_{1} & \Delta_{2} & x_{3} & x_{10} & x_{11} & x_{12} \\
x_{2} & x_{3} & \Delta_{3} & x_{13} & x_{14} & x_{15} \\
x_{7} & x_{10} & x_{13} & \Delta_{4} & x_{4} & x_{5} \\
x_{8} & x_{11} & x_{14} & x_{4} & \Delta_{5} & x_{6} \\
x_{9} & x_{12} & x_{15} & x_{5} & x_{6} & \Delta_{6}
\end{array}\right]_{6 \times 6}
$$

$\Lambda(t, N)$ has the following analytical expression:

$$
\Lambda(t, N)=\left[\begin{array}{llllll}
\Delta_{1} & \mu_{1} & \mu_{2} & \mu_{7} & \mu_{8} & \mu_{9} \\
\mu_{1} & \Delta_{2} & \mu_{3} & \mu_{10} & \mu_{11} & \mu_{12} \\
\mu_{2} & \mu_{3} & \Delta_{3} & \mu_{13} & \mu_{14} & \mu_{15} \\
\mu_{7} & \mu_{10} & \mu_{13} & \Delta_{4} & \mu_{4} & \mu_{5} \\
\mu_{8} & \mu_{11} & \mu_{14} & \mu_{4} & \Delta_{5} & \mu_{6} \\
\mu_{9} & \mu_{12} & \mu_{15} & \mu_{5} & \mu_{6} & \Delta_{6}
\end{array}\right]_{6 \times 6}
$$

From [1], we have the following analytical expressions for $\theta(N)$ and $w(N)$ :

$\theta(2 n)=\sum_{p=1}^{2 n} \sum_{q=1}^{2 n}\left|a_{p}, q\right|\left(1-\exp \left(-\left|a_{p}, q\right|\right) \quad\right.$ and $\theta(2 n-1)=\sum_{p=1}^{2 n-1} \sum_{q=1}^{2 n-1}\left|a_{p}, q\right|\left(1-\exp \left(-\left|a_{p}, q\right|\right)\right.$

$w(N)=1-\exp (-\theta(N))$, where $N=2 n$ or $N=2 n-1$ 
$B(N)$ is defined as a Linear combination of the Baseline Matrices associated with the Individual Constituent Matrix Shells, as follows:

$B(N)=\sum_{t=0}^{n-1}(w(N))^{t} B(t, N) \quad$, where $(w(N))^{0}=1$

Therefore, we have: $B(N)=\left(X(N) \otimes I_{2 \times 2}\right)+\left(\Lambda(N) \otimes \Sigma_{2 \times 2}\right)$

where, $\quad X(N)=\sum_{t=0}^{n-1}(w(N))^{t} X(t, N)$ and $\Lambda(N)=\sum_{t=0}^{n-1}(w(N))^{t} \Lambda(t, N)$

From [1], we have the following expression:

$$
S(j \mid t, N)=\left(X(t, N) \otimes I_{2 \times 2}\right)+\left(Y(j \mid t, N) \otimes \Sigma_{2 \times 2}\right) \quad, \text { where } j=1,2,3, \ldots \ldots \ldots, 32
$$

Therefore,

$S(j \mid t, N)=B(t, N)+D(j \mid t, N)$, where $D(j \mid t, N)=(Y(j \mid t, N)-\Lambda(t, N)) \otimes \Sigma_{2 \times 2}, j=1,2,3$,

The following relationship exists between the trace value of matrix $B(t, N)$ and that of the matrices $S(j \mid t, N)$ : $\forall j=1,2,3, \ldots \ldots . . ., 32$ we have:

$$
\operatorname{trace}(S(j \mid t, N))=\operatorname{trace}(B(t, N))=2 \operatorname{trace}(X(t, N))=2\left(\Delta_{1}+\Delta_{2}+\Delta_{3}+\Delta_{4}+\Delta_{5}+\Delta_{6}\right)
$$

we have, from [1], the Analytical Expression for the State-Interaction Matrix description of the Matrix Shell System $N=2 n$ or $N=2 n-1$, given as follows:

$S\left(j_{1}, j_{2}, \ldots . ., j_{n} \mid N\right)=\sum_{t=0}^{n-1}(w(N))^{t} S\left(j_{t+1} \mid t, N\right)$, where $(w(N))^{0}=1, \quad j_{1} \in I . S, j_{2} \in I . S, \ldots ., j_{n} \in I . S$

The index set $I . S$ is given as $I . S=\{1,2, \ldots . ., 31,32\}$

Therefore,

$S\left(j_{1}, j_{2}, \ldots \ldots, j_{n} \mid N\right)=B(N)+D\left(j_{1}, j_{2}, \ldots ., j_{n} \mid N\right)$ where we have:

$D\left(j_{1}, j_{2}, \ldots . ., j_{n} \mid N\right)=\sum_{t=0}^{n-1}(w(N))^{t} D\left(j_{t+1} \mid t, N\right) \quad$ and $\quad(w(N))^{0}=1, j_{1} \in I . S, j_{2} \in I . S, \ldots ., j_{n} \in I . S$ also, $D\left(j_{1}, j_{2}, \ldots . ., j_{n} \mid N\right)=\left(Y\left(j_{1}, j_{2}, \ldots ., j_{n} \mid N\right)-\Lambda(N)\right) \otimes \Sigma_{2 \times 2}$, and we have :

$Y\left(j_{1}, j_{2}, \ldots, j_{n} \mid N\right)=\sum_{t=0}^{n-1}(w(N))^{t} Y\left(j_{t+1} \mid t, N\right)$, where $(w(N))^{0}=1, j_{1} \in I . S, j_{2} \in I . S, \ldots ., j_{n} \in I . S$

Hence, we finally have the following Expression:

$S\left(j_{1}, j_{2}, \ldots . ., j_{n} \mid N\right)=\left(X(N) \otimes I_{2 \times 2}\right)+\left(Y\left(j_{1}, j_{2}, \ldots ., j_{n} \mid N\right) \otimes \Sigma_{2 \times 2}\right), j_{1} \in I . S, j_{2} \in I . S, \ldots ., j_{n} \in I . S$ and we have the following relationship associated with the trace values :

$\operatorname{trace}\left(S\left(j_{1}, j_{2}, \ldots ., j_{n} \mid N\right)\right)=\operatorname{trace}(B(N))=2 \operatorname{trace}(X(N))=\sum_{t=0}^{n-1}(w(N))^{t}\left[\sum_{p=1}^{6} 2 \Delta_{p}(t, N)\right]$,

where $j_{1} \in I . S, j_{2} \in I . S, \ldots ., j_{n} \in I . S$ 


\section{Determination of the most Stable Configuration pair(s) associated with the $A(t, N)$ Matrix Shell}

In this section, a formalism is presented to determine the most stable configuration pair(s) of a Matrix Shell $A(t, N)$, associated with an even type $(\mathrm{N}=2 \mathrm{n})$ or odd type $(\mathrm{N}=2 \mathrm{n}-1)$ Matrix Shell System.

- The issue of stability of the configuration pairs relative to each other, is dependant intrinsically on the precise numerical realization of the Fundamental matrix in the appropriate Matrix space and thereby, on the numerical realization of the Matrix Shell under consideration.

- In the presented formalism, the issue of relative stability of the configuration pairs is addressed through the eigenvalue analysis of their corresponding State-Interaction matrices, thereby; the Matrix Shell configurations constituting a dual pair are equally stable in context of the presented formalism.

- In context of the present formalism, one or more configuration pairs associated with a particular numerical realization of the Matrix Shell can satisfy the criterion of Maximum Stability.

- The Ordered Eigen spectrum of a Real, Symmetric Matrix $A_{n \times n}$, whose eigenvalues are $\lambda_{1}, \lambda_{2}, \ldots \ldots \ldots, \lambda_{n}$, is the set $O . E S\left(A_{n \times n}\right)=\left\{\lambda^{(1)}, \lambda^{(2)}, \ldots \ldots, \lambda^{(n)}\right\}$, where $\lambda^{(1)} \geq \lambda^{(2)} \geq \ldots \ldots . \geq \lambda^{(n)}$

- The issue of Stability, in context of the present formalism, is addressed in terms of Proximity of Ordered Eigen spectrum of the Individual Configuration pair State-Interaction matrices to the Ordered Eigen spectrum associated with the Baseline Matrix. The greater this spectral proximity of a configuration pair relative to another, the greater is its stability relative to the other configuration pair.

$$
\begin{aligned}
& O . E S(S(j \mid t, N))=\left\{\lambda_{1}(j), \lambda_{2}(j), \ldots \ldots \ldots, \lambda_{11}(j), \lambda_{12}(j)\right\}, \forall j \in I . S \\
& O . E S(B(t, N))=\left\{\lambda_{1}(b), \lambda_{2}(b), \ldots \ldots \ldots, \lambda_{11}(b), \lambda_{12}(b)\right\}
\end{aligned}
$$

We have the Proximity function defined as follows:

$$
P(S(j \mid t, N), B(t, N))=\exp \left(-\left[\left|\lambda_{1}(j)-\lambda_{1}(b)\right|+\left|\lambda_{2}(j)-\lambda_{2}(b)\right|+\ldots \ldots . .+\left|\lambda_{11}(j)-\lambda_{11}(b)\right|+\left|\lambda_{12}(j)-\lambda_{12}(b)\right|\right]\right) \quad \forall j \in I . S
$$

Therefore, the Most Stable Configuration pair 'c ', $c \in I . S$, satisfies the following condition :

$$
P(S(c \mid t, N), B(t, N))=\max (P(S(1 \mid t, N), B(t, N)), P(S(2 \mid t, N), B(t, N)), \ldots ., P(S(32 \mid t, N), B(t, N)))
$$

Determination of the Most Stable Configurations of the Matrix Shell System $N=2 n$ and $N=2 n-1$

Note: In the following scheme, it is assumed that each of the ' $\mathrm{n}$ ' constituent Matrix Shells $A(0, N), A(1, N), \ldots \ldots, A(n-1, N)$ corresponding to the Matrix Shell System $\mathrm{N}=2 \mathrm{n}$ or $\mathrm{N}=2 \mathrm{n}-1$, are numerically active (Chapter 1, [1]), i.e., each of them are in non-zero numerical realization.

In the general case involving one or more Matrix Shells in numerically inactive realizations, the presented scheme is to be applied taking into consideration only those constituent Matrix Shells that are present in numerically active realizations.

- The Matrix Shell System of type $\mathrm{N}=2 \mathrm{n}$ or the type $\mathrm{N}=2 \mathrm{n}-1$, both constituted by ' $\mathrm{n}$ ' Matrix Shells $A(0, N), A(1, N), \ldots \ldots, A(n-1, N)$, has the issue of its most Stable Configurations addressed in terms of the most stable configuration pair(s) associated with each of its constituent Matrix Shells.

- In determining the most stable configurations of the Matrix Shell system in a particular plausible numerical realization, consideration is restricted to the set of State-Interaction matrix descriptions of the Matrix Shell System, that arise out of linear combinations of only those State-Interaction matrix descriptions of the constituent Matrix Shells that satisfy the condition of maximum stability for the Individual constituent Matrix Shells, this set is denoted as $\left\{S\left(c_{1}, c_{2}, \ldots ., c_{n} \mid N\right)\right\}$ 
$\left\{S\left(c_{1}, c_{2}, \ldots, c_{n} \mid N\right)\right\}=\left\{S\left(j_{1}, j_{2}, \ldots, j_{n} \mid N\right): S\left(j_{1}, j_{2}, \ldots, j_{n} \mid N\right)=\sum_{t=0}^{n-1}(w(N)){ }^{t} S\left(c_{t+1} \mid t, N\right)\right\}$

$c_{1}, c_{2}, \ldots ., c_{n} \in I . S$, where "c $c_{1}$ " denotes the most stable configuration pair associated with the Matrix Shell $A(0, N)$, "c $c_{2}$ " denotes the most stable configuration pair associated with the Matrix Shell $A(1, N)$, ......, "c $\mathrm{c}_{\mathrm{n}}$ " denotes the most stable configuration pair associated with the Matrix Shell $A(n-1, N)$

Note: There can be more than one such ' $c$ ' index that satisfies the condition for maximum stability for a given Matrix Shell $A(t, N)$, the set $\left\{S\left(c_{1}, c_{2}, \ldots, c_{n} \mid N\right)\right\}$ consists of all possible linear combinations of individual Matrix shell State-Interaction Matrices, taking this degeneracy into consideration.

' $m_{1}$ ' be the number of State-Interaction matrices satisfying the maximum stability criterion for the Matrix Shell $A(0, N)$

' $m_{2}$ ' be the number of State-Interaction matrices satisfying the maximum stability criterion for the Matrix Shell $A(1, N)$

And finally, ' $m_{n}$ ' be the number of State-Interaction matrices satisfying the maximum stability criterion for the Matrix Shell $A(n-1, N)$

Therefore,

$$
\left|\left\{S\left(c_{1}, c_{2}, \ldots, c_{n} \mid N\right)\right\}\right|=m_{1} \times m_{2} \times \ldots \times m_{n}=\prod_{i=1}^{n} m_{i}
$$

Taking into account the duality aspect relating a configuration pair of a Matrix Shell to a State-Interaction matrix description, we have the following:

Total number of Possible Most Stable Configurations of a Matrix Shell System (N=2n Type or N=2n-1 Type) in an arbitrary plausible numerical realization $=2^{n}\left(m_{1} m_{2} \ldots . . . m_{n}\right)=2^{n}\left(\prod_{i=1}^{n} m_{i}\right)$

Therefore,

$$
\left|\left\{\left(v_{1} ; v_{2} ; \ldots, v_{n}\right)\right\}\right|=2^{n}\left(\prod_{i=1}^{n} m_{i}\right) \quad \ldots . .(\text { eqn. })
$$

we have the following:

$O . E S\left(S\left(j_{1}, j_{2}, \ldots ., j_{n} \mid N\right)\right)=\left\{\lambda_{1}\left(j_{1}, j_{2}, \ldots ., j_{n} \mid N\right), \lambda_{2}\left(j_{1}, j_{2}, \ldots ., j_{n} \mid N\right), \ldots \ldots, \lambda_{11}\left(j_{1}, j_{2}, \ldots ., j_{n} \mid N\right), \lambda_{12}\left(j_{1}, j_{2}, \ldots ., j_{n} \mid N\right)\right\}$

where $S\left(j_{1}, j_{2}, \ldots, j_{n} \mid N\right) \in\left\{S\left(c_{1}, c_{2}, \ldots, c_{n} \mid N\right)\right\}$

and $O \cdot E S(B(N))=\left\{\lambda_{1}(b, N), \lambda_{2}(b, N)\right.$ $\left.\lambda_{11}(b, N), \lambda_{12}(b, N)\right\}$

Therefore,

$$
\begin{aligned}
P\left(S\left(j_{1}, j_{2}, \ldots, j_{n} \mid N\right), B(N)\right)=\exp \left(-\left[\sum_{r=1}^{12}\left|\lambda_{r}\left(j_{1}, j_{2}, \ldots, j_{n} \mid N\right)-\lambda_{r}(b, N)\right|\right]\right) & \\
& \forall S\left(j_{1}, j_{2}, \ldots, j_{n} \mid N\right) \in\left\{S\left(c_{1}, c_{2}, \ldots ., c_{n} \mid N\right)\right\}
\end{aligned}
$$

In terms of the above Spectral proximity condition, the subset of the $\operatorname{set}\left\{S\left(c_{1}, c_{2}, \ldots, c_{n} \mid N\right)\right\}$, that satisfy the condition of maximum Spectral proximity, is denoted as $\left\{S\left(\psi_{1}, \psi_{2}, \ldots ., \psi_{n} \mid N\right)\right\}$

Therefore,

$\left\{S\left(\psi_{1}, \psi_{2}, \ldots ., \psi_{n} \mid N\right)\right\} \subseteq\left\{S\left(c_{1}, c_{2}, \ldots ., c_{n} \mid N\right)\right\}$, each element of the set $\left\{S\left(\psi_{1}, \psi_{2}, \ldots ., \psi_{n} \mid N\right)\right\}$ satisfies the following condition: 


$$
\begin{aligned}
& P\left(S\left(\psi_{1}, \psi_{2}, \ldots ., \psi_{n} \mid N\right), B(N)\right)=\max \left(P\left(S\left(j_{1}, j_{2}, \ldots, j_{n} \mid N\right), B(N)\right)\right), \\
& \text { over } \left.\forall S\left(j_{1}, j_{2}, \ldots ., j_{n} \mid N\right) \in\left\{S\left(c_{1}, c_{2}, \ldots ., c_{n} \mid N\right)\right\}\right)
\end{aligned}
$$

The set of Matrix Shell System Configurations that is associated with the set $\left\{S\left(\psi_{1}, \psi_{2}, \ldots ., \psi_{n} \mid N\right)\right\}$ is the set of all Configurations of the Matrix Shell System $(N=2 n$ or $N=2 n-1)$ that conform to the condition of maximum stability, it is denoted as $\left\{\left(\phi_{1} ; \phi_{2} ; \ldots . . ; \phi_{n}\right)\right\}$

Hence, the total No. of Configurations of the Matrix Shell System ( $N=2 n$ or $N=2 n-1)$ that satisfy the condition of maximum stability $=\left|\left\{\left(\phi_{1} ; \phi_{2} ; \ldots . . ; \phi_{n}\right)\right\}\right|=2^{n}\left(\left|\left\{S\left(\psi_{1}, \psi_{2}, \ldots ., \psi_{n} \mid N\right)\right\}\right|\right)$

\section{Case Studies based on Hypothetical Example Scenarios}

1) $N=4$ Matrix Shell System

Let $m_{1}=2$ and $m_{2}=2$; for the Hypothetical example let $c_{1}=3,5$ and $c_{2}=2,7$

From [Chapter 3, [1]], the Configuration pair pertaining to $A(0,4)$ Matrix Shell associated with $j=3$ : $(1,3,5 \mid 2, \overline{4}, 6),(\overline{1}, \overline{3}, \overline{5} \mid \overline{2}, 4, \overline{6})$

The Configuration pair pertaining to $A(0,4)$ Matrix Shell associated with $j=5$ :

$(1,3,5 \mid 2, \overline{4}, \overline{6}),(\overline{1}, \overline{3}, \overline{5} \mid \overline{2}, 4,6)$

The Configuration pair pertaining to $A(1,4)$ Matrix Shell associated with $j=2$ :

$(1,3,5 \mid \overline{2}, 4,6),(\overline{1}, \overline{3}, \overline{5} \mid 2, \overline{4}, \overline{6})$

The Configuration pair pertaining to $A(1,4)$ Matrix Shell associated with $j=7$ :

$(1,3,5 \mid \overline{2}, \overline{4}, 6),(\overline{1}, \overline{3}, \overline{5} \mid 2,4, \overline{6})$

Therefore:

$\left\{S\left(c_{1}, c_{2} \mid N=4\right)\right\}=\{S(3,2 \mid N=4), S(3,7 \mid N=4), S(5,2 \mid N=4), S(5,7 \mid N=4)\}$

For this Hypothetical sample let $\left\{S\left(\psi_{1}, \psi_{2} \mid N=4\right)\right\}=\{S(3,7 \mid N=4)\}$, hence, the set of Most Stable Configurations of the exemplified $N=4$ Matrix Shell System is given as follows:

$$
\begin{aligned}
& \left\{\left(\phi_{1} ; \phi_{2}\right)\right\}=\{(1,3,5,2, \overline{4}, 6 ; 1,3,5, \overline{2}, \overline{4}, 6),(1,3,5,2, \overline{4}, 6 ; \overline{1}, \overline{3}, \overline{5}, 2,4, \overline{6}), \\
& (\overline{1}, \overline{3}, \overline{5}, \overline{2}, 4, \overline{6} ; 1,3,5, \overline{2}, \overline{4}, 6),(\overline{1}, \overline{3}, \overline{5}, \overline{2}, 4, \overline{6} ; \overline{1}, \overline{3}, \overline{5}, 2,4, \overline{6})\}
\end{aligned}
$$

2) $N=6$ Matrix Shell System

Let $m_{1}=1, m_{2}=1$ and $m_{3}=3$; for the Hypothetical sample $c_{1}=5, c_{2}=16$ and $c_{3}=10,17,22$

The Configuration pair pertaining to $A(0,6)$ Matrix Shell associated with $j=5$ :

$$
(1,3,5 \mid 2, \overline{4}, \overline{6}),(\overline{1}, \overline{3}, \overline{5} \mid \overline{2}, 4,6)
$$


The Configuration pair pertaining to $A(1,6)$ Matrix Shell associated with $j=16$ :

$$
(\overline{1}, 3,5 \mid \overline{2}, \overline{4}, \overline{6}),(1, \overline{3}, \overline{5} \mid 2,4,6)
$$

The Configuration pair pertaining to $A(2,6)$ Matrix Shell associated with $j=10$ :

$$
(\overline{1}, 3,5 \mid \overline{2}, 4,6),(1, \overline{3}, \overline{5} \mid 2, \overline{4}, \overline{6})
$$

The Configuration pair pertaining to $A(2,6)$ Matrix Shell associated with $j=17$ :

$$
(1, \overline{3}, 5 \mid 2,4,6),(\overline{1}, 3, \overline{5} \mid \overline{2}, \overline{4}, \overline{6})
$$

The Configuration pair pertaining to $A(2,6)$ Matrix Shell associated with $j=22$ :

$$
(1, \overline{3}, 5 \mid \overline{2}, 4, \overline{6}),(\overline{1}, 3, \overline{5} \mid 2, \overline{4}, 6)
$$

Therefore,

$$
\left\{S\left(c_{1}, c_{2}, c_{3} \mid N=6\right)\right\}=\{S(5,16,10 \mid N=6), S(5,16,17 \mid N=6), S(5,16,22 \mid N=6)\}
$$

for this Hypothetical sample let :

$$
\left\{S\left(\psi_{1}, \psi_{2}, \psi_{3} \mid N=6\right)\right\}=\{S(5,16,10 \mid N=6), S(5,16,22 \mid N=6)\}
$$

hence, the set of Most Stable Configurations of the exemplified $N=6$ Matrix Shell System is given as follows:

$\left\{\left(\phi_{1} ; \phi_{2} ; \phi_{3}\right)\right\}=$

$\{(1,3,5,2, \overline{4}, \overline{6} ; \overline{1}, 3,5, \overline{2}, \overline{4}, \overline{6} ; \overline{1}, 3,5, \overline{2}, 4,6),(1,3,5,2, \overline{4}, \overline{6} ; \overline{1}, 3,5, \overline{2}, \overline{4}, \overline{6} ; 1, \overline{3}, \overline{5}, 2, \overline{4}, \overline{6})$,

$(1,3,5,2, \overline{4}, \overline{6} ; 1, \overline{3}, \overline{5}, 2,4,6 ; \overline{1}, 3,5, \overline{2}, 4,6),(1,3,5,2, \overline{4}, \overline{6} ; 1, \overline{3}, \overline{5}, 2,4,6 ; 1, \overline{3}, \overline{5}, 2, \overline{4}, \overline{6})$,

$(\overline{1}, \overline{3}, \overline{5}, \overline{2}, 4,6 ; \overline{1}, 3,5, \overline{2}, \overline{4}, \overline{6} ; \overline{1}, 3,5, \overline{2}, 4,6),(\overline{1}, \overline{3}, \overline{5}, \overline{2}, 4,6 ; \overline{1}, 3,5, \overline{2}, \overline{4}, \overline{6} ; 1, \overline{3}, \overline{5}, 2, \overline{4}, \overline{6})$,

$(\overline{1}, \overline{3}, \overline{5}, \overline{2}, 4,6 ; 1, \overline{3}, \overline{5}, 2,4,6 ; \overline{1}, 3,5, \overline{2}, 4,6),(\overline{1}, \overline{3}, \overline{5}, \overline{2}, 4,6 ; 1, \overline{3}, \overline{5}, 2,4,6 ; 1, \overline{3}, \overline{5}, 2, \overline{4}, \overline{6})$,

$(1,3,5,2, \overline{4}, \overline{6} ; \overline{1}, 3,5, \overline{2}, \overline{4}, \overline{6} ; 1, \overline{3}, 5, \overline{2}, 4, \overline{6}),(1,3,5,2, \overline{4}, \overline{6} ; \overline{1}, 3,5, \overline{2}, \overline{4}, \overline{6} ; \overline{1}, 3, \overline{5}, 2, \overline{4}, 6)$,

$(1,3,5,2, \overline{4}, \overline{6} ; 1, \overline{3}, \overline{5}, 2,4,6 ; 1, \overline{3}, 5, \overline{2}, 4, \overline{6}),(1,3,5,2, \overline{4}, \overline{6} ; 1, \overline{3}, \overline{5}, 2,4,6 ; \overline{1}, 3, \overline{5}, 2, \overline{4}, 6)$,

$(\overline{1}, \overline{3}, \overline{5}, \overline{2}, 4,6 ; \overline{1}, 3,5, \overline{2}, \overline{4}, \overline{6} ; 1, \overline{3}, 5, \overline{2}, 4, \overline{6}),(\overline{1}, \overline{3}, \overline{5}, \overline{2}, 4,6 ; \overline{1}, 3,5, \overline{2}, \overline{4}, \overline{6} ; \overline{1}, 3, \overline{5}, 2, \overline{4}, 6)$,

$(\overline{1}, \overline{3}, \overline{5}, \overline{2}, 4,6 ; 1, \overline{3}, \overline{5}, 2,4,6 ; 1, \overline{3}, 5, \overline{2}, 4, \overline{6}),(\overline{1}, \overline{3}, \overline{5}, \overline{2}, 4,6 ; 1, \overline{3}, \overline{5}, 2,4,6 ; \overline{1}, 3, \overline{5}, 2, \overline{4}, 6)\}$

\section{CONCLUSION}

The paper presents an approach to address the issue of determining the most stable (most preferred) configurations of a Matrix Shell system, and provides the necessary platform for further numerical/computational studies to understand the intricacies of the Matrix Shell Model framework, in particular, to develop an understanding on how the changes in the elements of the numerical matrix affect the spectral properties of the State-Interaction matrix descriptions and Baseline matrices, and thereby, the set of most preferred configurations of the Matrix Shell system.
The Matrix Shell Model formalism is an algebraic framework, hence, the approach taken in this paper to address the issue of determination of stability of Matrix Shell Configurations and Configurations of the Matrix Shell System, is purely algebraic without invoking other constraints that may be imposed when the framework is applied to a real world problem, in such situation, the approach may need to be appropriately modified or an alternate methodology need to be applied to suit the requirements/constraints imposed by the problem. 


\section{REFERENCES}

\section{Books}

[1]. Ghosh, Debopam, A Tryst with Matrices: The Matrix Shell Model Formalism, $24 b y 7$ Publishing, India.

[2]. Datta, B. N., Numerical Linear Algebra and Applications, SIAM

[3]. Graham, Alexander, Kronecker Products \& Matrix Calculus with Applications, Dover Publications, Inc.

[4]. Haykin, Simon, Neural Networks A Comprehensive Foundation, $2^{\text {nd }}$ Edition, Pearson Education, Inc.

[5]. Jordan, Thomas. F., Quantum Mechanics in Simple Matrix Form, Dover Publications, Inc.

[6]. Nakahara, Mikio, and Ohmi, Tetsuo, Quantum Computing: From Linear Algebra to Physical Realizations, CRC Press.

[7]. Pratihar, D. K., Soft Computing: Fundamentals and Applications, Alpha Science International Ltd.

[8]. Strang, Gilbert, Linear Algebra and its Applications, $4^{\text {th }}$ Edition, Cengage Learning

[9]. Sundarapandian, V., Numerical Linear Algebra, PHI Learning Private Limited

\section{Research Articles}

[10]. Brewer, J. W., Kronecker Products and Matrix Calculus in System Theory, IEEE Trans. on Circuits and Systems, 25, No.9, p 772-781 (1978)

[11]. Hardy, Lucien, Quantum Theory from Five Reasonable Axioms, arXiv: quant-ph/0101012v4 (2001)

[12]. Macklin, Philip A., Normal matrices for physicists, American Journal of Physics, 52, 513(1984)

[13]. Paris, Matteo G A, The modern tools of Quantum Mechanics : A tutorial on quantum states, measurements and operations, arXiv: 1110.6815v2 [ quant-ph ] (2012)

[14]. Roth, W. E., On Direct Product Matrices, Bull. Amer. Math. Soc., No. 40, p 461-468 (1944) 